\title{
Propuesta de un plan de mantenimiento preventivo para centrales de aire acondicionado
}

\author{
Wuilkild Pérez Dávila ${ }^{1}$ \\ Celemis Torres Robleto ${ }^{2}$ \\ Rubén Camacho Martínez ${ }^{3}$ \\ Wilfredo Van de Velde ${ }^{4}$
}

\section{RESUMEN}

Comprende los trabajos realizados en los equipos centrales de aire acondicionado en el centro hospitalario San Juan de Dios en la ciudad de Estelí, tales como los diagnósticos del estado de los equipos y su rutina de mantenimientos, las mediciones con respecto a las horas trabajadas por cada máquina y por el técnico especializado en su atención y los costos y beneficios que corresponderían a un cambio en la filosofía de mantenimiento al pasar del tipo correctivo al clasificado como preventivo. Todos estos estudios definieron las bases necesarias para una propuesta de un plan de mantenimiento preventivo que garantizara la vida útil de los aires centrales y que permitiera un control definido del funcionamiento de estos así como los análisis en cuanto a la calidad del servicio percibido por la población.

Palabras Clave: Mantenimiento, preventivo, centrales.

Recibido: 26 de agosto de 2015

Aceptado: 6 de octubre de 2015

1 UNAN-Managua/FAREM-Estelí. Correo Electrónico: wuilkildprz91@gmail.com

2 UNAN-Managua/FAREM-Estelí. Correo Electrónico: celdan21@hotmail.com

3 UNAN-Managua/FAREM-Estelí. Correo Electrónico: rubenantonioc@yahoo.com

4 UNAN-Managua FAREM-Estelí. Correo Electrónico: wilfredo.van.de.velde@gmail.com 


\section{Proposal of a preventive maintenance plan for central air conditioning}

\section{SUMARY}

It includes the job done in the central air conditioners of the hospital San Juan de Dios in the city of Estelí, such as the diagnostics of the equipment and the routine of maintenance, measurements with respect to the hours worked by each machine and by the specialized technician in his work, the costs and benefits that would correspond to a change in the maintenance philosophy from a corrective form to a preventive one. These studies defined the necessary basis for a proposal of a preventive maintenance plan that will guarantee the useful life of the central airs, this will allow a defined function of these analyzes and control as to the quality of service perceived by the population.

Keywords: Maintenance, Preventive, plants. 


\section{INTRODUCCIÓN}

El presente documento está basado en la elaboración de un plan de gestión de mantenimiento preventivo para las unidades centrales de aire acondicionado del Hospital San Juan de Dios en la ciudad de Estelí.

Esta investigación contempla el diagnóstico del sistema de mantenimiento implementado en los equipos centrales de aire acondicionado en dicho centro médico con el propósito de diseñar una propuesta que sirva de base para un plan de mantenimiento preventivo para estas unidades y garantizar de esta manera un servicio de calidad para la población en general.

Para la conservación de la infraestructura y el equipamiento, el papel del mantenimiento consiste en:

1. Asegurar la correcta operación e incrementar la confiabilidad aplicando estrategias fundamentales de mantenimiento para las maquinas y equipos a través de la realización de la planeación, organización, dirección y control.

2. Aplicar métodos y técnicas de conservación con miras a lograr una disminución en las incidencias de fallas imprevistas para mantener niveles altos de disponibilidad de la capacidad instalada, garantizando las óptimas condiciones de bienestar para el paciente o usuario.

En estas páginas están registradas las metas propuestas, las razones por las se ha realizado este trabajo, un marco teórico que permite la comprensión de conceptos en base al campo en que se han realizado los estudios, y una explicación detallada del tipo de investigación y análisis que se desarrollaron para el diseño del manual de mantenimiento preventivo adaptado a las necesidades de dicho centro hospitalario.

\section{MATERIALES Y METODOS}

Localización de la investigación

Esta investigación se realizó en las instalaciones del Hospital San Juan de Dios, localizado en el kilómetro 148 carretera norte en la ciudad de Estelí, siendo su posición geográfica la siguiente: $13^{\circ} 05^{\prime}$ latitud norte y $86^{\circ} 21^{\prime}$ longitud oeste, tiene una altura de 800 metros sobre el nivel del mar, cabecera municipal del departamento de Estelí, a 147 km de la ciudad Capital Managua, Nicaragua.

\section{Tipo de Estudio}

Esta investigación se define como cuali-cuantitativa. Cuantitativa por que abordamos aspectos económicos que determinaron los beneficios de la implementación de la prevención en lugar de la corrección en cuanto a mantenimiento y de qué manera los costos planteados pueden ser distribuidos a lo largo del año y cualitativa porque debemos tomar como punto guía el rendimiento optimo de los quipos, sus características especificas y funcionamiento así como las características y factores de un buen mantenimiento de las centrales de aire acondicionado y de qué manera logra esto brindar un buen servicio a las y los pacientes.

\section{Población y muestra}

Puesto que contamos con tres poblaciones distintas para la aplicación de nuestros instrumentos investigativos, (Equipos Centrales de aire acondicionado, Técnicos y Jefe del departamento de mantenimiento y Pacientes), hemos determinado nuestra muestra de la siguiente manera: Serán analizados los tiempos de todas las centrales de aire siendo un total de 15 equipos todas en estado activo, las entrevistas y trabajo directo será implementado con el jefe del departamento de mantenimiento y el técnico a cargo del mantenimiento de las centrales de aire acondicionado por ser el único de los demás técnicos que cuenta con la preparación especializada en climatización, y una muestra de 30 pacientes, seleccionados al azar, para determinar la calidad del servicio y la climatización de las diferentes 
aéreas del hospital a través de encuestas aplicadas en los diferentes turnos (matutino, vespertino, nocturno).

\section{Etapas de la investigación}

La investigación se realizó directamente en el campo de aplicación para desarrollar mejor las mediciones de tiempo y funcionamiento necesarias en los equipos y trabajar de la mano junto con el personal a cargo del mantenimiento de estos.

Los pasos a desarrollar en la investigación para el cumplimiento de los objetivos propuestos son los siguientes:

1. Se involucró directamente en las actividades de mantenimiento para identificar las acciones involucradas directamente en el procedimiento de corrección y el tiempo que se toman el técnico en la realización de esta tarea. Además de la implementación de mediciones de tiempo y consumo energético.

2. Se recopiló documentación en base a los manuales de fábrica de los equipos, las hojas de vida de cada central e información bibliográfica y recopilada en las entrevistas y encuestas para determinar de qué manera puede ser aplicada la teoría en el diseño de un manual preventivo.

3. Se diseñó una propuesta de plan de mantenimiento preventivo y se ajustaran detalles.

4. Estudios de comparación serán realizados para determinar si el nuevo plan de mantenimiento preventivo brinda los resultados económicos esperados a través del balance costo-beneficio.

5. Se evaluó en reuniones con el jefe de mantenimiento y técnico encargado del aire acondicionado para la crítica constructiva del plan de mantenimiento preventivo y determinar si se ajusta las necesidades y si es aplicable con los recursos económicoshumanos con los que cuenta la institución.

\section{Técnicas de recolección de la información}

\section{a. Revisión de la bibliografía.}

Fueron estudiados libros de referencia que permitieron conocer herramientas para las medidas de tiempos y diseño de plan de mantenimiento.

\section{b. Observación del proceso de mantenimiento.}

La observación y participación directa en el proceso de mantenimiento sirvió para mostrar de qué manera estaba funcionando la institución y como este mismo proceso podía ser optimizado con el mantenimiento preventivo.

\section{c. Medición de tiempos.}

Se realizaron para la obtención de información en base a los procedimientos a modo de hojas de control y diagramas analíticos, todo esto en el periodo de 4 semanas para registrar la variación del funcionamiento de los equipos que hubo y que fueron base para nuestro estudio.

\section{d. Realización de encuestas y entrevistas.}

Para la evaluación de la calidad en el servicio y percepción de la climatización de las diferentes salas se realizó una encuesta (Anexos) a los pacientes, dicha encuesta está compuesta de 6 preguntas y se realizó tanto a pacientes del género masculino como del femenino en diferentes situaciones y áreas de recuperación. En cuanto a la entrevista aplicada al jefe del departamento de mantenimiento y técnico encargado de los aires sirvió para identificar las principales consecuencias producidas por el fallo de estas centrales y a la vez para determinar la capacitación del personal para la implementación de un correcto mantenimiento.

\section{Tratamiento de la información}

Después de que fueron realizados los procedimientos mencionados anteriormente se procedió al análisis y tratamiento de la información recolectada, a través de la digitalización de la información recolectada en las entrevistas a modo de conclusiones conjuntas. Fueron 
implementados los Diagramas de Pareto y de la Ruta Critica como resultado de los estudios implementados en las mediciones de tiempo. Para la elaboración del documento se utilizó, en la plataforma operativa de Windows, Word 2013, para el procesamiento de datos referentes al control de costos se implementó siempre en este mismo sistema operativo el programa Excel 2013.

\section{RESULTADOS}

Puesto que parte de nuestra investigación era el involucramiento directo en las actividades del departamento de mantenimiento relacionadas con las centrales de aire acondicionado nos integramos como parte de este departamento de la institución sanitaria, de esta manera obtuvimos como resultado un diagnostico de dicha área administrativa y pudimos apreciar su organización clasificada como tipo jerárquica siendo el principal y único responsable el ingeniero y jefe, procedimientos de contingentes compuesto principalmente por ordenes de trabajo, bitácora de incidentes y reportes, planeaciones de tareas semanales que abarcan las áreas de caldera, equipos médicos, aires acondicionado, electricidad, utilería y limpieza, horario de los técnicos equivalente 8 horas diarias a C\$ 37.50 por hora y la capacitación referente a cada una de las áreas que atienden en lo que determinamos que todos cuentan con lo necesario, inventarios disponibles en el caso del área de utilería y limpieza más no así las áreas de equipos médicos ni aires acondicionados y el tipo de herramientas con las que dan respuesta a las diferentes fallas o correcciones de los equipos tanto médicos como institucionales.

Al ser parte del departamento de mantenimiento tuvimos participación directa en el proceso de mantenimiento rutinario de los equipos centrales de aire acondicionado, al participar de este proceso aplicamos el método de observación para de este modo reconocer las partes de los aires que cumplen con el ciclo de transmisión de calor en las diferentes áreas donde están instaladas las centrales, las herramientas y materiales implementados en el proceso correctivo de fallas en los equipos con lo que logramos recoger información de costos de cada uno de estos para diseñar un plan de costos anual de mantenimiento preventivo utilizando también el número de horas que los equipos trabajan diariamente y como estas horas varían según la temporada de festividades como Navidad y Semana Santa, conseguimos clasificar las fallas más comunes y el número de veces que se presentaban en el año para la estructuración del diagrama de Pareto, y los procedimientos y tiempo real de cada una de las tareas involucradas en el proceso son la base de información para el diseño de un modelo de modo de ruta critica. A continuación presentaremos estos dos diagramas y la tabla de costos anuales como fruto de nuestra investigación.

\section{Diagrama de Pareto}

\begin{tabular}{|c|c|c|c|}
\hline $\begin{array}{l}\text { Fallas equipos } \\
\text { centrales de aire } \\
\text { acondicionado }\end{array}$ & $\begin{array}{c}\mathbf{N}^{\circ} \text { de fallas } \\
\text { Anuales }\end{array}$ & $\%$ & $\begin{array}{c}\% \\
\text { Acumulado }\end{array}$ \\
\hline Bandas & 60 & 81.08 & 81.08 \\
\hline Rejillas & 6 & 8.10 & 89.18 \\
\hline $\begin{array}{l}\text { Herramientas del } \\
\text { técnico }\end{array}$ & 3 & 4.05 & 93.23 \\
\hline Conductores eléctricos & 3 & 4.05 & 97.28 \\
\hline Fuga de refrigerante & 2 & 2.70 & 99.98 \\
\hline Total & 74 & $99.98 \%$ & $99.98 \%$ \\
\hline
\end{tabular}

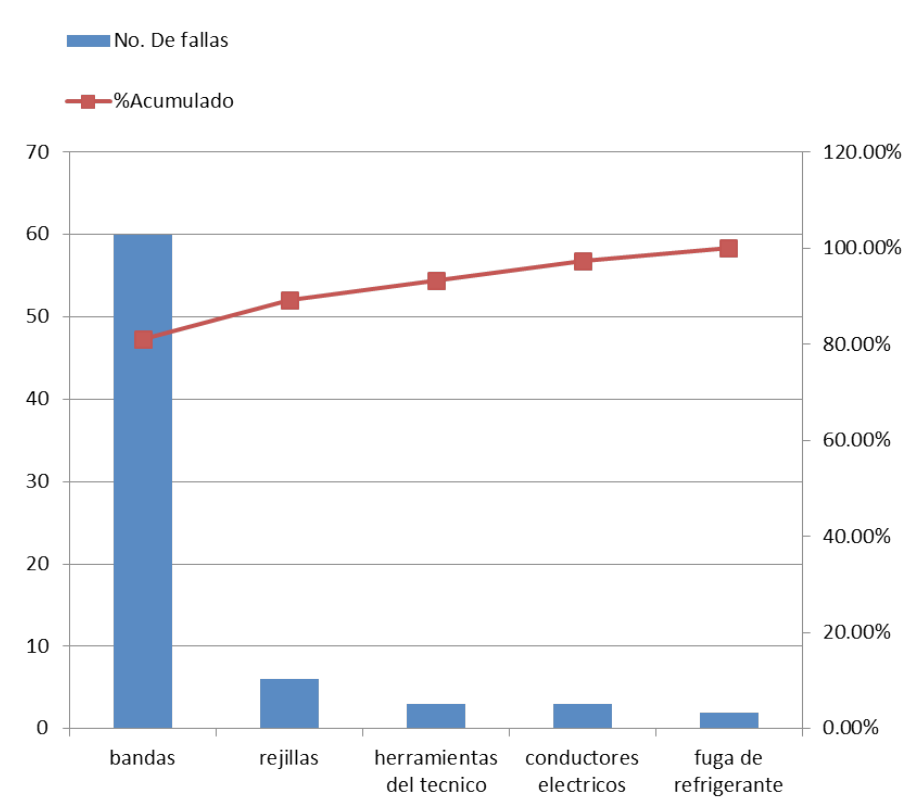




\section{Modelo de Ruta Crítica}

Es un proceso administrativo de planificación, programación, ejecución y control de todas y cada una de las actividades componentes de un proyecto que debe desarrollarse dentro de un tiempo crítico y al costo mínimo. Actualmente existen 15 equipos de aire acondicionado en las diferentes áreas del Hospital San Juan de Dios, del tipo de unidad paquete de 12 $1 / 2$ toneladas de refrigeración. El motivo de realizar la propuesta de mantenimiento preventivo bajo el método de la ruta critica, es con la finalidad de realizar una propuesta mejor en cuestión de tiempo para reducir los costos y reducir el tiempo de ejecución.

\begin{tabular}{|c|c|c|c|}
\hline $\mathbf{N}^{\circ}$ & Actividad & $\begin{array}{c}\text { Tiempo } \\
\text { (minutos) }\end{array}$ & Antecedentes \\
\hline 1 & $\begin{array}{l}\text { Revisión de vibraciones y } \\
\text { ruidos extraños del equipo } \\
\text { puesto en marcha }\end{array}$ & 5 & 0 \\
\hline 2 & $\begin{array}{l}\text { Revisión y ajuste de anclaje } \\
\text { del equipo }\end{array}$ & 30 & 1 \\
\hline 3 & $\begin{array}{l}\text { Revisión de instalación } \\
\text { eléctrica del tablero general }\end{array}$ & 20 & 1 \\
\hline 4 & $\begin{array}{l}\text { Revisión de tablero derivado } \\
\text { del equipo }\end{array}$ & 10 & 3 \\
\hline 5 & Desarmado del equipo & 60 & 4 \\
\hline 6 & $\begin{array}{l}\text { Revisión y ajuste de } \\
\text { chumaceras y cojinetes del } \\
\text { motor }\end{array}$ & 30 & 2 \\
\hline 7 & $\begin{array}{l}\text { Revisión y ajuste de aspas y } \\
\text { rotor del ventilador }\end{array}$ & 15 & 5 \\
\hline 8 & $\begin{array}{l}\text { Revisión de nivel de aceite y } \\
\text { del compresor }\end{array}$ & 5 & 7 \\
\hline 9 & $\begin{array}{l}\text { Carga de aceite del } \\
\text { compresor }\end{array}$ & 20 & 8 \\
\hline 10 & $\begin{array}{l}\text { Revisión de presiones de } \\
\text { aceite y protecciones del } \\
\text { comprensor }\end{array}$ & 20 & 9 \\
\hline 11 & $\begin{array}{l}\text { Revisión de niveles de } \\
\text { refrigerante R-22 y fugas }\end{array}$ & 15 & 10 \\
\hline 12 & $\begin{array}{l}\text { Revisión y ajuste de } \\
\text { resistencia del cárter }\end{array}$ & 20 & 11 \\
\hline 13 & $\begin{array}{l}\text { Revisión y ajuste de válvulas } \\
\text { de expansión y solenoides }\end{array}$ & 20 & 6 \\
\hline 14 & $\begin{array}{l}\text { Revisión y ajuste de } \\
\text { termostato de bajo ambiente }\end{array}$ & 10 & 13 \\
\hline
\end{tabular}

\begin{tabular}{|c|c|c|c|}
\hline 15 & $\begin{array}{l}\text { Limpieza y purga de } \\
\text { serpentines del condensador } \\
\text { y unidad manejadora }\end{array}$ & 120 & 14 \\
\hline 16 & $\begin{array}{l}\text { Limpieza y purga de trampas } \\
\text { de desagüe }\end{array}$ & 60 & 15 \\
\hline 17 & $\begin{array}{l}\text { Limpieza de rejillas de } \\
\text { inyección y retorno }\end{array}$ & 150 & 16 \\
\hline 18 & Limpieza de filtros metálicos & 90 & 7 \\
\hline 19 & Cambio de filtros metálicos & 60 & 18 \\
\hline 20 & $\begin{array}{l}\text { Limpieza de charola de } \\
\text { condensadores }\end{array}$ & 15 & 19 \\
\hline 21 & $\begin{array}{l}\text { Alineación de bandas y } \\
\text { poleas }\end{array}$ & 20 & 20 \\
\hline 22 & $\begin{array}{l}\text { Limpieza de piedras } \\
\text { disecantes }\end{array}$ & 30 & 15 \\
\hline 23 & Cargar de refrigerante R-22 & 15 & 11,21 \\
\hline 24 & $\begin{array}{l}\text { Lubricación de piezas } \\
\text { requeridas }\end{array}$ & 30 & 22 \\
\hline 25 & $\begin{array}{l}\text { Revisión de amperaje y } \\
\text { voltaje entre fases }\end{array}$ & 10 & 24 \\
\hline 26 & $\begin{array}{l}\text { Limpieza y apriete de } \\
\text { conexiones en tablero de } \\
\text { control }\end{array}$ & 15 & 25 \\
\hline 27 & Armado del equipo & 60 & 26 \\
\hline 28 & Arranque y pruebas & 20 & $12,17,23,28$ \\
\hline
\end{tabular}

Sellama red a la representación gráfica de las actividades que muestran sus eventos, secuencias, interrelaciones y la Ruta Critica, del mantenimiento preventivo de las unidades tipo paquete de aire acondicionado.

Como observaremos en la red siguiente podemos determinar que la ruta critica de del evento 1 pasando por el 2, 6, 13, 14, 15, 16, 17 hasta llegar al 28. Lo que, calculado a través de las holguras, determina que la duración máxima que se puede retrasar en su inicio sin que esto afecte el tiempo estimado del proceso es de 445 minutos o 7 horas con 26 minutos, por maquina.

De esta manera quedan determinados los pasos más importantes a seguir en el mantenimiento preventivo de las unidades y las que no pueden ser ignoradas al momento de una evaluación por parte del técnico. Además de contar con el tiempo más Óptimo. 


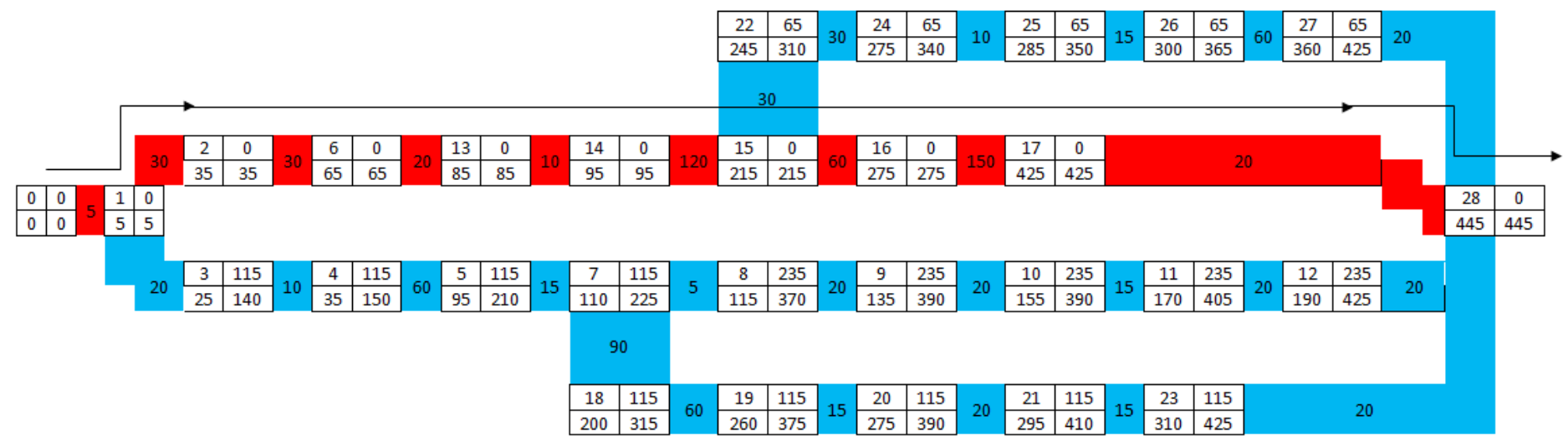

\begin{tabular}{|c|c|c|c|c|c|c|c|c|c|c|c|c|}
\hline & Enero & Febrero & Marzo & Abril & Mayo & Junio & Julio & Agosto & Septiembre & Octubre & Noviembre & Diciembre \\
\hline $\begin{array}{l}\text { Volumen de } \\
\text { Produccion (H-M) }\end{array}$ & 5,040 & 5,100 & 5,130 & 5,175 & 5,115 & 5,088 & 5,100 & 5,070 & 5,040 & 4,950 & 5,010 & 5,250 \\
\hline C fijo de M.O & 28,500 & 28,500 & 28,500 & 28,500 & 28,500 & 28,500 & 28,500 & 28,500 & 28,500 & 28,500 & 28,500 & 28,500 \\
\hline $\begin{array}{l}\text { Costo fijo de } \\
\text { materiales }\end{array}$ & 1,500 & 1,500 & 1,500 & 1,500 & 1,500 & 1,500 & 1,500 & 1,500 & 1,500 & 1,500 & 1,500 & 1,500 \\
\hline $\begin{array}{l}\text { C mano de obra } \\
\text { (Mant prev. Gen) }\end{array}$ & 0 & 0 & 9,300 & 0 & 0 & 9,300 & 0 & 0 & 9,300 & 0 & 0 & 9,300 \\
\hline $\begin{array}{l}\text { C Materialea (Mant } \\
\text { prev. Gen) }\end{array}$ & 0 & 0 & 1,300 & 0 & 0 & 1,300 & 0 & 0 & 1,300 & 0 & 0 & 1,300 \\
\hline $\begin{array}{l}\text { C Total (Mant prev. } \\
\text { Gen) }\end{array}$ & 0 & 0 & 10,600 & 0 & 0 & 10,600 & 0 & 0 & 10,600 & 0 & 0 & 10,600 \\
\hline $\begin{array}{l}\text { Matenimiento } \\
\text { especializado }\end{array}$ & 75,000 & & & & & & & & & & & \\
\hline $\begin{array}{l}\text { Costos totales de } \\
\text { M.O }\end{array}$ & 28,500 & 28,500 & 37,800 & 28,500 & 28,500 & 37,800 & 28,500 & 28,500 & 37,800 & 28,500 & 28,500 & 37,800 \\
\hline $\begin{array}{l}\text { Costos totales de } \\
\text { materiales }\end{array}$ & 1,500 & 1,500 & 2,800 & 1,500 & 1,500 & 2,800 & 1,500 & 1,500 & 2,800 & 1,500 & 1,500 & 2,800 \\
\hline $\begin{array}{l}\text { Costos variables } \\
\text { totales }\end{array}$ & 75,000 & 0 & 0 & 0 & 0 & 0 & 0 & 0 & 0 & 0 & 0 & 0 \\
\hline Costos fijos totales & 30,000 & 30,000 & 40,600 & 30,000 & 30,000 & 40,600 & 30,000 & 30,000 & 40,600 & 30,000 & 30,000 & 40,600 \\
\hline Costos totales & 105,000 & 30,000 & 40,600 & 30,000 & 30,000 & 40,600 & 30,000 & 30,000 & 40,600 & 30,000 & 30,000 & 40,600 \\
\hline
\end{tabular}

Como resultado de las encuestas realizadas a los pacientes obtuvimos los siguientes porcentajes, los cuales serán abarcados con diagramas para su mejor comprensión en cuanto al como es percibida la calidad del servicio por parte de los usuarios.

La primera pregunta es:

\section{¿Es usted un paciente frecuente en el hospital?}

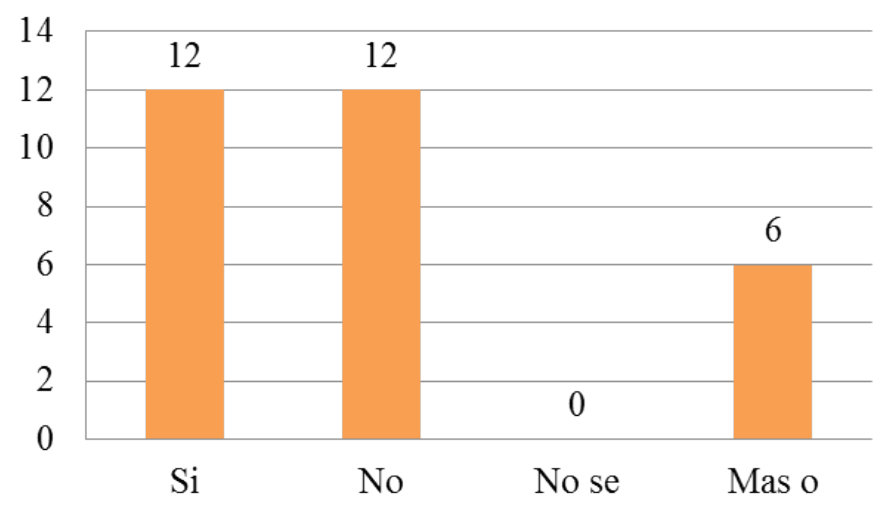


Tuvimos resultados variables como corresponde, al ser escogidos pacientes al azar encontramos que un $40 \%$ eran pacientes frecuentes, otro $40 \%$ no visitaban constantemente el hospital y un $20 \%$ más o menos

En nuestra pregunta número dos consultamos:

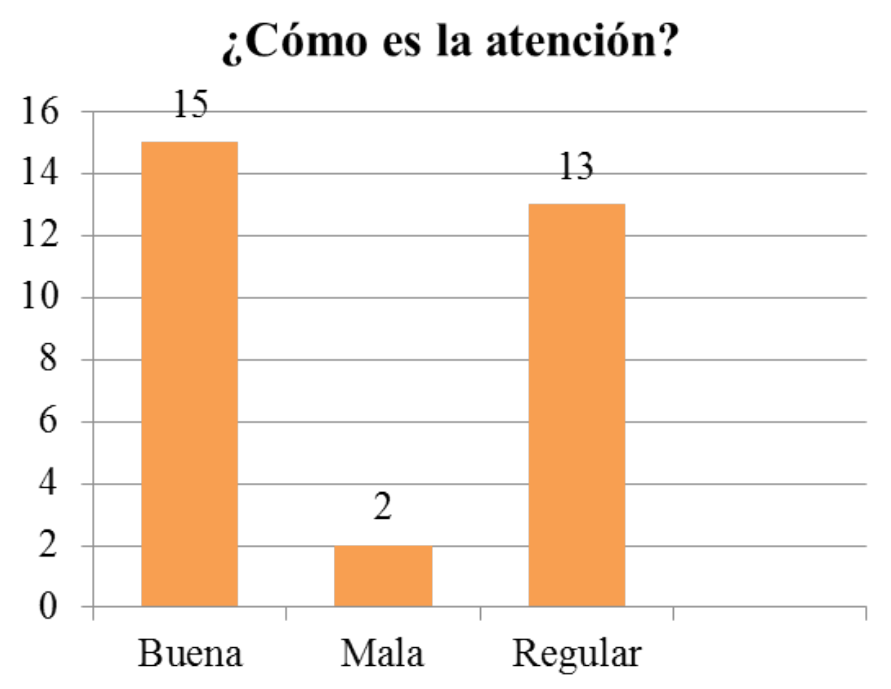

Como podemos apreciar, la percepción en cuanto a la calidad en la atención obtuvimos un 50\% de respuesta positivas, un $6.66 \%$ de valoración negativa y un $43.33 \%$ que opina que es regular.

Continuando con la encuesta, quisimos saber el tiempo reglar que en tardaban en ser atendidos los pacientes, a lo que respondieron a la pregunta:

¿Cuánto tiempo esta en la sala de espera?

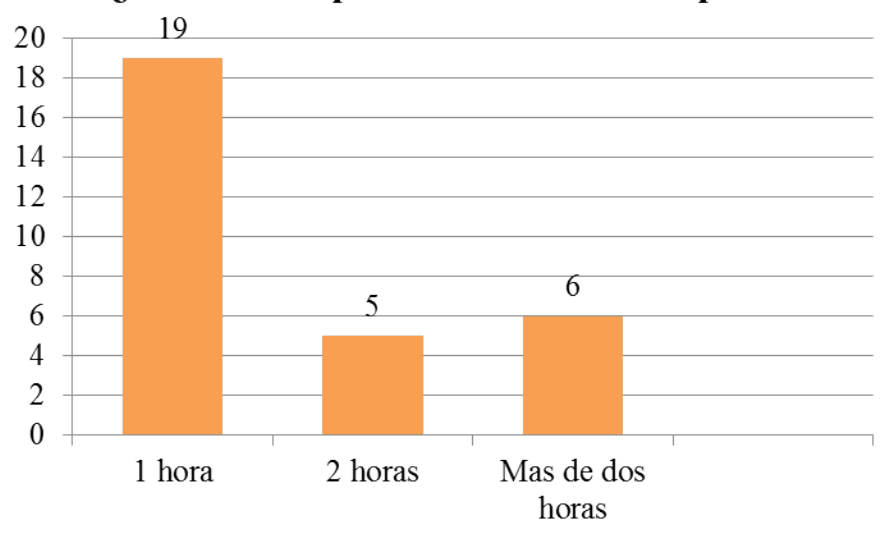

Como podemos observar un $63.33 \%$ considera que la atención por parte del personal médico llego en un hora después de haber arriba al centro de salud, aunque $16.66 \%$ han sido atendidos en dos horas y un $20 \%$ han sido atendidos en más de dos horas, lo que consideramos bastante realista.

Por lo que la siguiente pregunta nos lleva a consultar sobre las condiciones de esa espera:

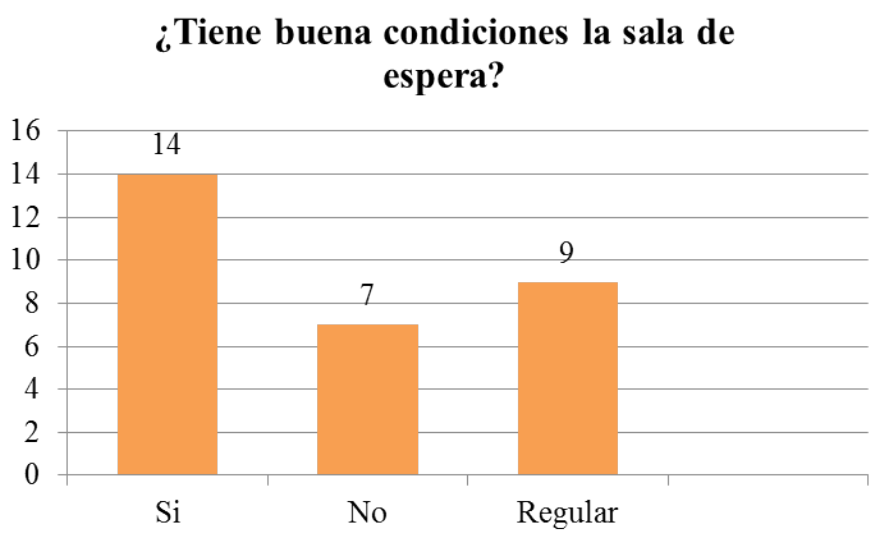

Un $46.66 \%$ valora como optimas las condiciones de la sala de espera, un $23.33 \%$ cree que estas condiciones son malas, y un $30 \%$ determina que son regulares.

Ya que estábamos evaluando las condiciones, debíamos indagar sobre la temperatura a lo que respondieron a la pregunta:

\section{¿Hay una buena temperatura en la sala} de espera?

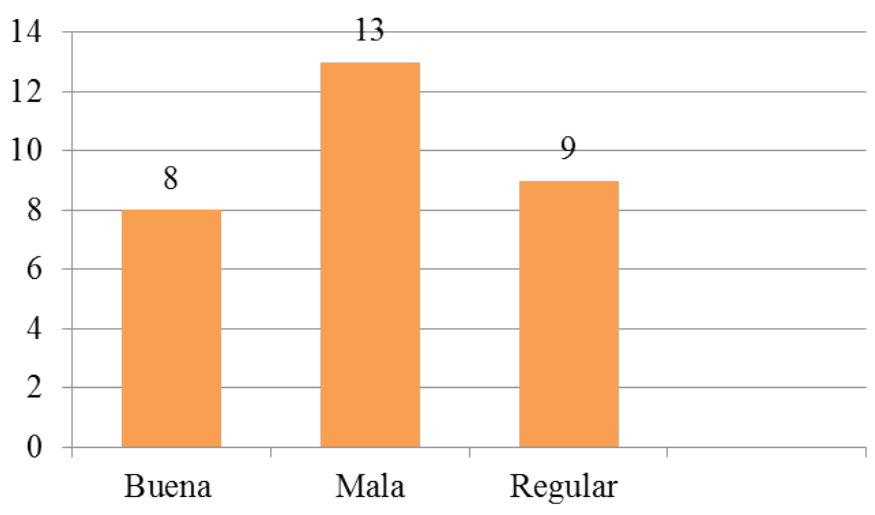


Un $26.66 \%$ cree que es buena, sin embargo es notorio el $43.33 \%$ no cree que la temperatura en la sala de espera sea la adecuada para un centro hospitalario y que debe ser mejorada para garantizar el confort de sus usuarios, podemos arriesgarnos a decir que el $30 \%$ que considera que es regular apoyarían la idea de mejora en este aspecto. Sin embargo en la siguiente pregunta a:

\section{¿Es importante tener una temperatura confortable en la sala de espera?}

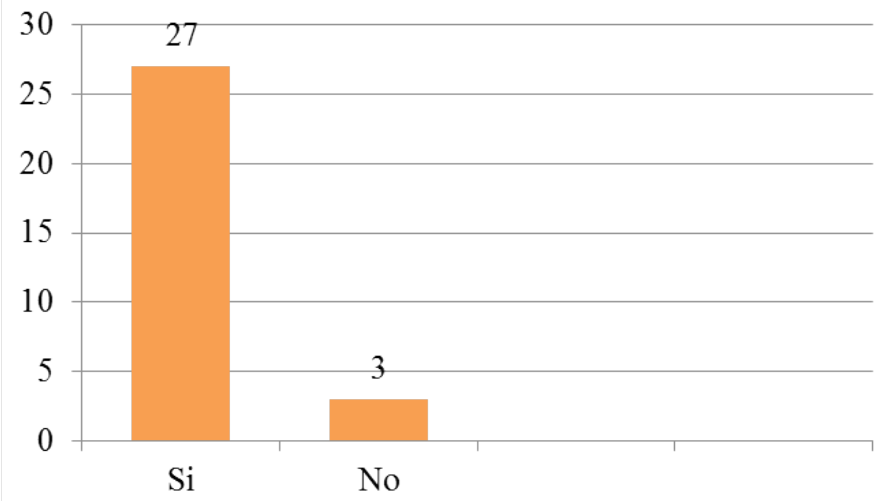

El 90\% considera que la temperatura en un aspecto importante en la sala de espera, y un $10 \%$ no lo ve de esta manera.

Como conclusión a las respuestas obtenidas en las encuestas podemos decir que la temperatura es un aspecto que necesita ser mejorado debido al tiempo que invierten los usuarios en las salas del centro hospitalario, esto nos ayudó significativamente a conocer de qué manera es percibida la calidad en el servicio.

\section{CONCLUSIONES}

El plan de mantenimiento para el Hospital San Juan de Dios, Estelí, es limitado, ya que se trabaja con hojas de rutina de mantenimiento y hojas de control de equipos para las centrales de aire, esto demuestra que no hay un plan de mantenimiento para dichas unidades centrales.

Durante las mediciones de tiempo pudimos crear un registro y en base al historial del trabajo logramos documentar los volúmenes de producción (hora trabajadas por las máquinas) en el año. Esta información junto con los datos de plantilla y costos de materiales nos permitieron la planificación anual del plan de mantenimiento preventivo.

A través de las encuestas logramos identifica la calidad del servicio percibida por la población y con la entrevista recopilamos información que nos sirvió de base para nuestro trabajo documental. A la vez con la participación directa en el proceso de mantenimiento de algunos de los equipos adquirimos conocimientos en cuanto al funcionamiento del ciclo de refrigeración, partes de las centrales y herramientas empleadas en el mantenimiento. Con esto logramos establecer una rutina con estándares de tiempo para el proceso de mantenimiento preventivo en cada uno de los equipos.

Al concluir con nuestro trabajo podemos ver la importancia que tiene el aplicar mantenimiento preventivo a las centrales de aire acondicionado ya que este garantiza un óptimo funcionamiento y permite que la institución brinde un servicio de excelente calidad a sus usuarios, así como también pudimos darnos cuenta lo importante que es contar con un plan de mantenimiento y cumplir con cada una de las indicaciones para que con esto se facilite el uso de estos equipos por parte de los técnicos.

Podemos decir que cumplimos satisfactoriamente con nuestros objetivos y que las metas planteadas se han visto realizadas, como prueba de esto queda el modelo de un Plan de Mantenimiento Preventivo como propuesta para los técnicos de mantenimiento, y lograr de esta manera los ahorros que los cálculos y presupuestos garantizan.

\section{RECOMENDACIONES}

Las siguientes recomendaciones son resultado de nuestra investigación. 
Se aconseja emplear un mantenimiento total cada tres años debido a que según la empresa aunque el equipo no muestre falla es recomendable para evitar algún deterioro por el uso en alguna pieza del aire, a su vez ayuda a mantener un registro del esta físico del aire.

La temperatura que debe de manejarse en las salas generales es de $25^{\circ}$ Celsius de esta manera se garantiza el confort para los usuarios y un óptimo desempeño para los quipos. Exentos de esta recomendación están los aires implementados en los quirófanos pues por desarrollar un trabajo directamente séptico necesitan una temperatura de $20^{\circ}$ Celsius lo que no afecta el desempeño de los aires puesto que se encuentra entre su rango de operaciones. Estas indicaciones están basadas en los estándares de la norma 100713-2005.

El mantenimiento preventivo propuesto en nuestro plan, debe ser realizado cada 3 meses, y debe ser revisado en caso de alguna falla inesperada para la reformulación de los presupuestos. El tiempo determinado es el mismo indicado por su manual de fábrica brindado por la compañía representante.

\section{BIBLIOGRAFÍA CONSULTADA}

Bravo Silva, R. (1989). Admisnitracion de Mantenimiento Industrial. San Jose, CR: Editorial UNED.

Carnicer, E. (2001). Aire Acondicionado. Madrid: Paraninfo.

Clima Proyecto. (s.f.). Paquetes de Aire Acondicionado. Obtenido de www.paquetesdeaireacondicionado. com/aire-acondicionado-tipo-paquete.html

Criollo, R. G. (1998). Estudio del Trabajo. Mexico D.F: Mc. Graw Hill.

Fuentes., I. A. (5 de Septiembre de 2014). Mantenimiento Preventivo Aires Acondicionados. (R. Camacho, Entrevistador)

Grijalbo Mondadori. (1997). Gran Diccionario Enciclopedico Ilustrado. Barcelona: Grijalbo S.A.

Madrigal R., M., \& Rosales, S. A. (1998). La productividad en el mantenimiento industrial. Mexico D.F: Compañia Editorial Continental S.A. Normalización, A. E. (2005). Patente $n^{\circ}$ UNE 1007132005. España. 